\title{
Magnetic reconnection driven by filament eruption in the 7 June 2011 event
}

\author{
L. van Driel-Gesztelyi ${ }^{1,2,3}$, D. Baker ${ }^{1}$, T. Török ${ }^{4}$, E. Pariat ${ }^{2}$, L. M. \\ Green $^{1}$, D. R. Williams ${ }^{1}$, J. Carlyle ${ }^{1}$, G. Valori ${ }^{2}$, P. Démoulin ${ }^{2}$, S. A. \\ Matthews $^{1}$, B. Kliem ${ }^{5}$ and J.-M. Malherbe ${ }^{2}$ \\ ${ }^{1}$ University College London, Mullard Space Science Laboratory, Holmbury St. Mary, Dorking, \\ Surrey RH5 6NT, UK \\ ${ }^{2}$ LESIA, Observatoire de Paris, CNRS, UPMC, Université Paris Diderot, 5 place Jules \\ Janssen, 92190 Meudon, France;email: Lidia.vanDriel@obspm.fr \\ ${ }^{3}$ Konkoly Observatory, Hungarian Academy of Sciences, Budapest, Hungary \\ ${ }^{4}$ Predictive Science Inc., 9990 Mesa Rim Rd., Suite 170, San Diego, CA 92121, USA \\ ${ }^{5}$ Institut für Physik und Astronomie, Universität Potsdam, Karl-Liebknecht-Str. 24-25, 14476 \\ Potsdam, Germany
}

\begin{abstract}
During an unusually massive filament eruption on 7 June 2011, SDO/AIA imaged for the first time significant EUV emission around a magnetic reconnection region in the solar corona. The reconnection occurred between magnetic fields of the laterally expanding CME and a neighbouring active region. A pre-existing quasi-separatrix layer was activated in the process. This scenario is supported by data-constrained numerical simulations of the eruption. Observations show that dense cool filament plasma was re-directed and heated in situ, producing coronal-temperature emission around the reconnection region. These results provide the first direct observational evidence, supported by MHD simulations and magnetic modelling, that a large-scale re-configuration of the coronal magnetic field takes place during solar eruptions via the process of magnetic reconnection.
\end{abstract}

Keywords. MHD, instabilities, Sun: activity, magnetic fields, coronal mass ejections (CMEs), filaments, methods: numerical, data analysis

A spectacular solar eruption occurred on 7 June 2011 observed by the Solar Dynamic Observatory's Atmospheric Imaging Assembly (SDO/AIA: Lemen et al. 2012). The CME originated in a complex of three adjacent active regions (ARs, see Figure 1) in the southwestern quadrant and carried an unusually massive erupting filament in its core. We carried out a multiwavelength analysis of the event. Using SDO/HMI data we computed the magnetic topology, determining the locations of quasi-separatrix layers in the threeAR complex. We also carried out data-constrained MHD simulations of the eruption.

We found that the strong lateral expansion of the erupting magnetic structure led to flux pile-up, current sheet formation/intensification, and magnetic reconnection along a pre-existing quasi-separatrix layer in the three-AR complex. The onset of reconnection first became apparent in the SDO/AIA images when downward flowing dense, cool filament plasma, originally contained within the erupting flux rope, was re-directed towards a neighbouring active region, tracing the change of large-scale magnetic connectivity. Williams et al. (2013) estimated a lower limit of the electron density of the redirected plasma to be $10^{10} \mathrm{~cm}^{-3}$, at least one order of magnitude larger than the typical coronal density. As a result of this unusually high density around the reconnection region, direct plasma heating took place there. The most prominent brightening was seen in the AIA $171 \AA$ waveband $\left(6.3 \times 10^{5} \mathrm{~K}\right)$.

These SDO observations provide one of the first direct imaging observations of magnetic reconnection in the solar atmosphere. Furthermore, a combination of observations, 


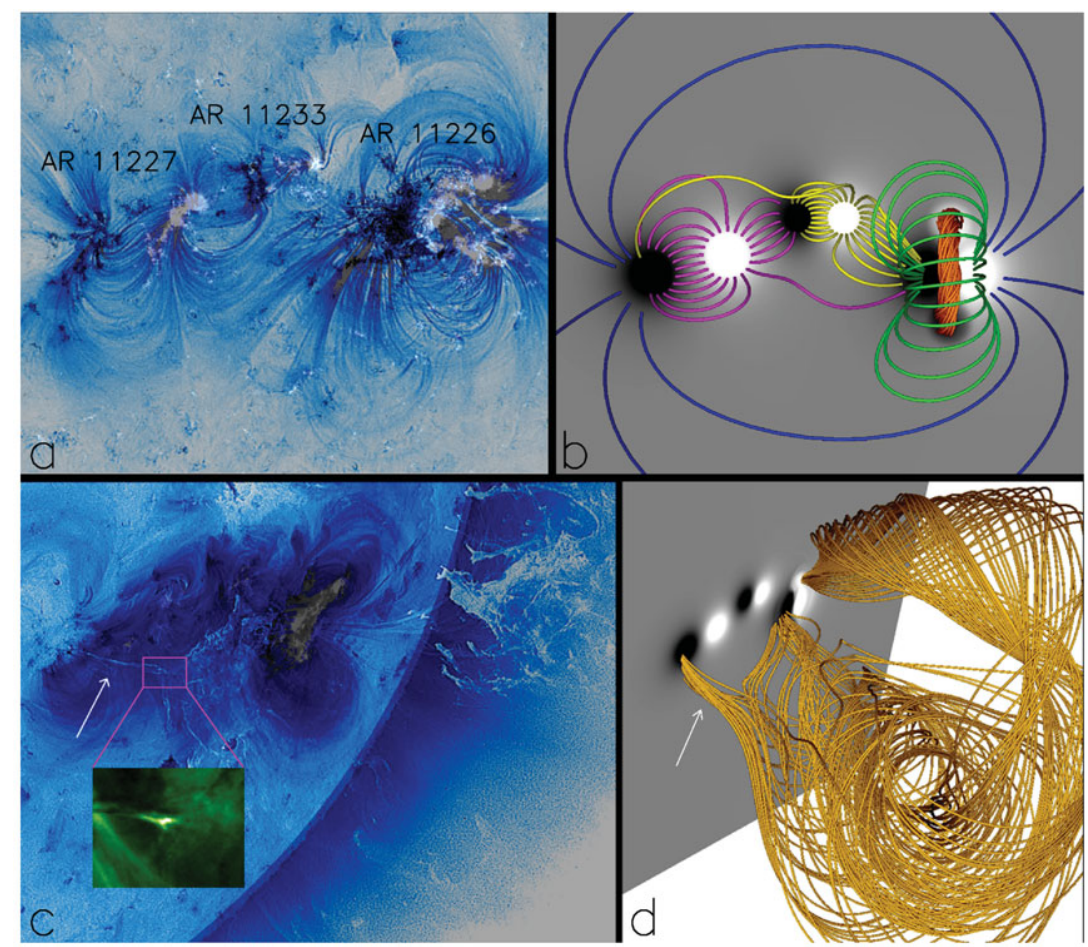

Figure 1. Observations and MHD numerical simulation of the magnetic configuration and the eruption. (a) SDO/AIA $171 \AA$ reverse colour scale image over a co-aligned SDO/HMI magnetic field map of the three neighbouring active regions on 3 June 2011 and (b) corresponding magnetic configuration in the simulation. Black/white indicate negative/positive magnetic polarity, the pre-eruption magnetic flux rope in the rightmost active region is shown in gold. (c) SDO/AIA $171 \AA$ reversed-colour image and (d) a simulation snapshot during the CME eruption on 7 June, by when the active regions have rotated close to the solar limb. The new connections, formed by magnetic reconnection between the magnetic flux rope erupting from AR 11226 and magnetic field lines of AR 11227, are indicated by white arrows in (c) and (d). The inset in (c) is a magnification of the in-situ heated bright reconnection region.

magnetic modelling, and MHD simulations (Figure 1) provides evidence that, during the expansion of a CME's magnetic structure, instantaneous magnetic reconnection can occur with ambient magnetic field leading to large-scale restructuring. For more details see van Driel-Gesztelyi et al. (2013).

\section{Acknowledgements}

The authors acknowledge funding from the European Commission's Seventh Framework Programme under the grant agreement No. 284461 (eHEROES project) and the Hungarian Research grant OTKA K-081421. TT was supported by NASA's HTP, LWS, SR\&T programs and NSF (AGS-1249270). LMG is grateful for a Royal Society Fellowship, and JC for a joint UCL-MPI Impact PhD Studentship. BK acknowledges support by the DFG.

\section{References}

Lemen, J. R., Title, A. M., Akin, D. J., et al., 2012, Solar Phys. 275, 17

van Driel-Gesztelyi, L., Baker, D., Török, T., Pariat, E., Green, L. M., Williams, D. R., Carlyle, J., Valori, G., Démoulin, P., Matthews, S. A., Kliem, B., \& Malherbe, J.-M. 2013, ApJ, submitted.

Williams, D. R., Baker, D., \& van Driel-Gesztelyi, L. 2013, ApJ, 764, 165 